\title{
The Lattice Structures of Approximation Operators Based on L-Fuzzy Generalized Neighborhood Systems
}

\author{
Qiao-Ling Song $\mathbb{D}^{1},{ }^{1}$ Hu Zhao $\mathbb{D},{ }^{1}$ Juan-Juan Zhang $\mathbb{D},{ }^{1}$ A. A. Ramadan $\mathbb{D}^{2},{ }^{2}$ \\ Hong-Ying Zhang $\mathbb{D}^{3}$, and Gui-Xiu Chen $\mathbb{D}^{4}$ \\ ${ }^{1}$ School of Science, Xi'an Polytechnic University, Xi'an 710048, China \\ ${ }^{2}$ Department of Mathematics, Faculty of Science, Beni-Suef University, Beni-Suef, Egypt \\ ${ }^{3}$ School of Mathematics and Statistics, Xi'an Jiaotong University, Xi'an 710049, China \\ ${ }^{4}$ School of Mathematics and Statistics, Qinghai Normal University, Xining 810008, China \\ Correspondence should be addressed to Hu Zhao; zhaohu@xpu.edu.cn
}

Received 9 February 2021; Revised 6 March 2021; Accepted 13 March 2021; Published 12 April 2021

Academic Editor: Heng Liu

Copyright (c) 2021 Qiao-Ling Song et al. This is an open access article distributed under the Creative Commons Attribution License, which permits unrestricted use, distribution, and reproduction in any medium, provided the original work is properly cited.

Following the idea of $L$-fuzzy generalized neighborhood systems as introduced by Zhao et al., we will give the join-complete lattice structures of lower and upper approximation operators based on $L$-fuzzy generalized neighborhood systems. In particular, as special approximation operators based on $L$-fuzzy generalized neighborhood systems, we will give the complete lattice structures of lower and upper approximation operators based on $L$-fuzzy relations. Furthermore, if $L$ satisfies the double negative law, then there exists an order isomorphic mapping between upper and lower approximation operators based on $L$-fuzzy generalized neighborhood systems; when $L$-fuzzy generalized neighborhood system is serial, reflexive, and transitive, there still exists an order isomorphic mapping between upper and lower approximation operators, respectively, and both lower and upper approximation operators based on $L$-fuzzy relations are complete lattice isomorphism.

\section{Introduction}

Pawlak $[1,2]$ defined the rough set theory to address the vagueness and granularity of information systems and data analysis. Many scholars worked on this theory and applied it to various fields [3-10]. The classical rough set theory is based on partition or equivalent relation. Thus, classical rough set theory has been extended to binary relation-based rough sets [11-14] and covering-based rough sets [15-19].

Fuzzy set theory is also an important mathematical tool to study uncertainty. Nowadays, there have been many branches of fuzzy mathematics, such as fuzzy algebra, fuzzy topology, and fuzzy logic [20-31]. Particularly, fuzzy rough set theory is an important branch, which can handle more complicated uncertain problems since it has the advantages of both fuzzy set and rough set [22, 32-38]. Furthermore, replacing the unit interval $[0,1]$ with a complete lattice $L$ as the range of the membership function [39], the more general $L$-fuzzy rough sets further extend the theoretical framework and application range of classic rough sets [31, 40-46]. Fuzzy rough sets have a variety of forms due to the different approaches of fuzzification. It is easily observed that the rough sets based on L-fuzzy relations and L-fuzzy coverings are the two most well-known L-fuzzy rough sets.

Recently, the notion of generalized neighborhood systems is proposed and used to define a theory of rough set, called generalized neighborhood system-based rough sets [45, 47-49]. In [49, 50], Zhao and Li gave an axiomatic characterization on generalized neighborhood system-based rough sets. Hence, it is natural to establish fuzzy general rough sets by fuzzifying them, respectively. Quite recently, by fuzzifying the notion of generalized neighborhood systems, Zhao and $\mathrm{Li}[49,50]$ established a rough set model based on $L$-fuzzy generalized neighborhood systems. It was proved that this model brought the fuzzy relation-based rough set model, fuzzy covering-based rough set model, and generalized neighborhood system-based rough set models under a unified framework. However, the lattice structures of approximation 
operators based on $L$-fuzzy generalized neighborhood systems were not studied. Following this idea, a natural problem arises: can the lattice structures of approximation operators based on $L$-fuzzy generalized neighborhood systems be given?

In the present paper, we study the lattice structures of approximation operators based on $L$-fuzzy generalized neighborhood systems (resp., $L$-fuzzy relations) and give the relationship between lower and upper approximation operators based on $L$-fuzzy generalized neighborhood systems (resp., $L$-fuzzy relations).

The contents of this paper are organized as follows. In Section 2, we recall some notions and notations used in this paper. In Section 3, the lattice structures of approximation operators based on L-fuzzy generalized neighborhood system operators are given. In Section 4, the lattice structures of lower and upper approximation operators based on $L$-fuzzy relations are discussed. In Section 5, we make a conclusion.

\section{Preliminaries}

In this section, we recall some basic notions and notations used in this paper.

A commutative quantale is a pair $(L, *)$, where $L$ is a complete lattice with respect to a partial order $\leq$ on it, with the top (resp., bottom) element 1 (resp., 0), and * is a commutative semigroup operation on $L$ such that $a * \vee_{j \in J} b_{j}=\vee_{j \in J}\left(a * b_{j}\right)$ for all $a \in L$ and $\left\{b_{j} \mid j \in J\right\} \subseteq L$. $(L, *)$ is said to be integral if the top element 1 is the unique unit in the sense of $1 * a=a$ for all $a \in L$.

In this paper, if not otherwise specified, $L=(L, *)$ is always assumed to be an integral, commutative quantale. Since the binary operation $*$ distributes over arbitrary joins, the function $a *(-): L \longrightarrow L$ has a right adjoint $a \longrightarrow(-): L \longrightarrow L$ given by $a \longrightarrow b=\vee\{c \in L: a * c \leq b\}$. We collect here some basic properties of the binary operations $*$ and $\longrightarrow[51-53] . L$ is said to satisfy the double negation law if for any $a \in L,(a \longrightarrow 0) \longrightarrow 0=a$, and we use $\neg a$ to denote $a \longrightarrow 0$. If $L$ satisfies the double negative law, then (1) $a \longrightarrow b=\neg b \longrightarrow \neg a$; $(a \longrightarrow b)=\neg(a * \neg b) ;(3) \neg\left(\wedge_{j \in J} a_{j}\right)=\vee_{j \in J}\left(\neg a_{j}\right)$.

\section{Proposition 1}

(1) $a \longrightarrow b=1 \Longleftrightarrow a \leq b$.

(2) $a *(a \longrightarrow b) \leq b$.

(3) $a \longrightarrow(b \longrightarrow c)=(a * b) \longrightarrow c=b \longrightarrow(a \longrightarrow c)$.

(4) $a \leq(a \longrightarrow b) \longrightarrow b$.

(5) $\left(\vee_{j \in J} a_{j}\right) \longrightarrow b=\wedge_{j \in J}\left(a_{j} \longrightarrow b\right)$.

(6) $a \longrightarrow\left(\wedge_{j \in J} b_{j}\right)=\wedge_{j \in J}\left(a \longrightarrow b_{j}\right)$.

(7) $\forall a \in L, a=\wedge_{b \in L}((a \longrightarrow b) \longrightarrow b)$.

Let the universe of discourse $X$ be an arbitrary nonempty set. We call a function $A: X \longrightarrow L$ as an $L$-fuzzy set in $X$. We use $L^{X}$ to denote the set of all $L$-fuzzy sets in $X$ and call it the $L$-fuzzy power set on $X$. For a crisp subset $A \subset X$, let $\chi_{A}$ be the characteristic function, i.e., $\chi_{A}(x)=1$ if $x \in A$ and $\chi_{A}(x)=0$ if $x \notin A$. The characteristic function $\chi_{A}$ of a subset $A \subseteq X$ can be regarded as an $L$-fuzzy set in $X$. We make no difference between a constant $L$-fuzzy set and its value since no confusion will arise.

The operators $\vee, \wedge, *, \longrightarrow$ on $L$ can be translated onto $L^{X}$ in a point-wise. That is, for any $A, B, A_{t}(t \in T) \in L^{X}$,

$$
\begin{aligned}
\left(\bigvee_{t \in T} A_{t}\right)(x) & =\vee_{t \in T} A_{t}(x), \\
\left(\bigwedge_{t \in T} A_{t}\right)(x) & =\bigwedge_{t \in T} A_{t}(x), \\
(A * B)(x) & =A(x) * B(x), \\
(A \longrightarrow B)(x) & =A(x) \longrightarrow B(x), \\
(\neg A)(x) & =\neg A(x) .
\end{aligned}
$$

Let $A, B$ be $L$-fuzzy set in $X$. The subsethood degree [20] of $A, B$, denoted by $S(A, B)$, is defined by $S(A, B)=$ $\wedge_{x \in X}(A(x) \longrightarrow B(x))$. $S(A, B)$ represents the semantics " $A$ is contained in $B . "$

Let $A, B$ be $L$-fuzzy set in $X$. The intersection degree [32] of $A, B$, denoted by $I(A, B)$, is defined by $I(A, B)=\vee_{x \in X}(A(x) * B(x)) . I(A, B)$ represents the semantics " $A$ and $B$ have intersection."

The lemma below collects some properties of subsethood degree and intersection degree. They can be found in many literatures such as $[6,22-24,30,32,52]$.

Lemma 1. Let $A, B, C, D, A_{t}(t \in T) \in L^{X}$. Then,
(S1) $S(A, B)=1 \Longleftrightarrow A \leq B$.
(S2) $A \leq B \Rightarrow S(A, C) \geq S(B, C)$ and $S(C, A) \leq S(C, B)$.
(S3) $S(A, B) * S(C, D) \leq S(A * C, B * D)$.
(S4) $S(A, B) * S(B, C) \leq S(A, C)$.
(S5) $S\left(A, \wedge_{t \in T} A_{t}\right)=\wedge_{t \in T} S\left(A, A_{t}\right)$.
(S6) $S(A, \alpha \longrightarrow B)=\alpha \longrightarrow S(A, B)$.
(S7) $S(A, \alpha * B) \geq \alpha * S(A, B)$.
(I1) $I(A, B)=I(B, A)$.
(I2) $I\left(A, \vee_{t \in T} A_{t}\right)=\vee_{t \in T} I\left(A, A_{t}\right)$.
(I3) $I(A, \alpha * B)=\alpha * I(A, B)$.
(I4) $I(A, \alpha \longrightarrow B) \leq \alpha \longrightarrow I(A, B)$.
(IS) $I(A, B) \longrightarrow \alpha=S(A, B \longrightarrow \alpha)$.

Next, we recall the notions of $L$-fuzzy generalized neighborhood systems and rough approximation operators based $L$-fuzzy generalized neighborhood systems.

Definition 1 (see $[49,50]$ ). Let $X$ be a set; the function $N: X \longrightarrow L^{L^{X}}$ is called an $L$-fuzzy generalized neighborhood system operator on $X$, if for any $x \in X$, $\vee_{C \in L^{X}} N(x)(C)=1$. Usually, $N(x)$ is called an $L$-fuzzy generalized neighborhood system of $x$ and $N(x)(C)$ is interpreted as the degree of that $C$ which is a neighborhood of $x$. The condition " $\vee_{C \in L^{X}} N(x)(C)=1$ " is a lattice-valued interpretation of " $N(x)$ is nonempty." In particular,

(1) An L-fuzzy generalized neighborhood system operator $N$ is said to be serial (denoted by SE), if for any $x \in X$ and $C \in L^{X}, N(x)(C) \leq \vee_{y \in X} C(y)$. 
(2) An $L$-fuzzy generalized neighborhood system operator $N$ is said to be reflexive (denoted by RE), if for any $x \in X$ and $C \in L^{X}, N(x)(C) \leq C(x)$.
(3) An $L$-fuzzy generalized neighborhood system operator $N$ is said to be transitive (denoted by TR), if for any $x \in X$ and $C \in L^{X}$,

$$
N(x)(C) \leq \underset{V \in L^{x}}{\vee}\left[N(x)(V) * \underset{y \in X}{\wedge}\left(V(y) \longrightarrow \underset{V_{y} \in L^{X}}{\vee}\left(N(y)\left(V_{y}\right) * S\left(V_{y}, C\right)\right)\right)\right] .
$$

Definition 2 (see $[49,50])$. Let $N: X \longrightarrow L^{L^{X}}$ be an $L$-fuzzy generalized neighborhood system operator. Then, for each
$A \in L^{X}$, the lower and upper approximation operators $\underline{N}(A)$ and $\bar{N}(A)$ are defined as follows:

$$
\begin{aligned}
& \underline{N}(A)(x)=\underset{C \in L^{X}}{\vee}(N(x)(C) * S(C, A)), \\
& \bar{N}(A)(x)=\underset{C \in L^{X}}{\wedge}(N(x)(C) \rightarrow I(C, A)) .
\end{aligned}
$$

Finally, we recall the notions of $L$-fuzzy relations and rough approximation operator-based $L$-fuzzy relations.

Definition 3 (see [36, 37]). An $L$-fuzzy relation on $X$ is a mapping $R: X \times X \longrightarrow L$. For any $(x, y) \in X \times X, R(x, y)$ is interpreted as the related degree between $x$ and $y$. Furthermore,

(1) $R$ is said to be serial if for all $x \in X, \vee_{y \in X} R(x, y)=1$.

(2) $R$ is said to be reflexive if for all $x \in X, R(x, x)=1$.

(3) $R$ is said to be transitive if for all $x, y, z \in X$, $R(x, y) * R(y, z) \leq R(x, z)$.

Definition 4 (see $[36,37]$ ). Let $R: X \times X \longrightarrow L$ be an $L$-fuzzy relation on $X$. Then, for any $A \in L^{X}$, the lower and upper approximation operators $\underline{R}(A)$ and $\bar{R}(A)$ are defined as follows:

$$
\begin{aligned}
& \underline{R}(A)(x)=\bigwedge_{y \in X}(R(x, y) \longrightarrow A(y)), \\
& \bar{R}(A)(x)=\bigvee_{y \in X}(R(x, y) * A(y)) .
\end{aligned}
$$

Lemma 2 (see [50]). Let $R: X \times X \longrightarrow L$ be an L-fuzzy relation on $X$. Define an L-fuzzy generalized neighborhood system operator $N_{R}$ as follows: for any $x \in X$ and $A \in L^{X}$,

$$
N_{R}(x)(A)= \begin{cases}1, & \text { if } A=R(x,-) \\ 0, & \text { otherwise. }\end{cases}
$$

Then, $\underline{N_{R}}(B)=\underline{R}(B)$ and $\overline{N_{R}}(B)=\bar{R}(B)$ for any $B \in L^{X}$.

\section{The Lattice Structures of Approximation Operators Based on $L$-Fuzzy Generalized Neighborhood Systems}

In this section, we will study the lattice structures of lower and upper approximation operators based on $L$-fuzzy generalized neighborhood systems.

Let

$$
\mathbf{L}-\operatorname{FGNS}(\mathbf{X})=\left\{N: X \longrightarrow L^{L^{X}} \mid \forall x \in X, \underset{C \in L^{X}}{\vee} N(x)(C)=1\right\},
$$

be the family of all $L$-fuzzy generalized neighborhood system operators on $X$, and let

$$
\begin{aligned}
\text { SE - LFGNS }(\mathbf{X}) & =\mathbf{L}-\operatorname{FGNS}(\mathbf{X}) \cap \mathbb{A}, \\
\operatorname{RE}-\operatorname{LFGNS}(\mathbf{X}) & =\mathbf{L}-\operatorname{FGNS}(\mathbf{X}) \cap \mathbb{B}, \\
\text { TR }-\operatorname{LFGNS}(\mathbf{X}) & =\mathbf{L}-\mathbf{F G N S}(\mathbf{X}) \cap \mathbb{C} .
\end{aligned}
$$

be the family of all serial, reflexive, and transitive $L$-fuzzy generalized neighborhood system operators on $X$, respectively, where

$$
\begin{aligned}
& \mathbb{A}=\left\{N: X \rightarrow L^{L^{X}} \mid \forall x \in X, C \in L^{X}, N(x)(C) \leq \underset{y \in X}{\vee} C(y)\right\}, \\
& \mathbb{B}=\left\{N: X \rightarrow L^{L^{X}} \mid \forall x \in X, C \in L^{X}, N(x)(C) \leq C(x)\right\}, \\
& \mathbb{C}=\left\{\begin{array}{c}
N: X \rightarrow L^{L^{X}} \mid \forall x \in X, C, \in L^{X}, N(x)(C) \\
\left.\leq \underset{V \in L^{X}}{\vee}\left[N(x)(V) * \underset{y \in X}{\wedge}\left(V(y) \rightarrow \underset{V_{y} \in L^{X}}{\vee}\left(N(y)\left(V_{y}\right) * S\left(V_{y}, C\right)\right)\right)\right]\right\} .
\end{array}\right.
\end{aligned}
$$


Let $\mathbf{L A O}(\mathbf{X})=\left\{N: L^{X} \longrightarrow L^{X} \mid N \in \mathbf{L}-\mathbf{F G N S}(\mathbf{X})\right\}$ and $\operatorname{UAO}(\mathbf{X})=\left\{\bar{N}: L^{X} \longrightarrow L^{X} \mid N \in \mathbf{L}-\mathbf{F G N S}(\mathbf{X})\right\} \quad$ be the family of all lower and upper approximation operators on $X$, respectively. Furthermore, let

$$
\begin{aligned}
& \operatorname{SE}-\mathbf{L A O}(\mathbf{X})=\left\{\underline{N}: L^{X} \longrightarrow L^{X} \mid N \in \operatorname{SE}-\operatorname{LFGNS}(\mathbf{X})\right\}, \\
& \operatorname{SE}-\operatorname{UAO}(\mathbf{X})=\left\{\bar{N}: L^{X} \longrightarrow L^{X} \mid N \in \operatorname{SE}-\operatorname{LFGNS}(\mathbf{X})\right\},
\end{aligned}
$$

be the family of all serial lower and upper approximation operators on $X$, respectively. Let RE-LAO $(\mathbf{X})=\left\{\underline{N}: L^{X} \longrightarrow L^{X} \mid N \in\right.$ RE - LFGNS $\left.(\mathbf{X})\right\}$ and RE$\operatorname{UAO}(\mathbf{X})=\left\{\bar{N}: L^{X} \longrightarrow L^{X} \mid N \in \operatorname{RE}-\operatorname{LFGNS}(\mathbf{X})\right\}$ be the family of all reflexive lower and upper approximation operators on $X$, respectively. Let TR - LAO $(\mathbf{X})=\left\{\underline{N}: L^{X} \longrightarrow L^{X} \mid N \in\right.$ TR - LFGNS $\left.(\mathbf{X})\right\}$ and TR$\mathbf{U A O}(\mathbf{X})=\left\{\bar{N}: L^{X} \longrightarrow L^{X} \mid N \in T R-\operatorname{LFGNS}(\mathbf{X})\right\}$ be the family of all transitive lower and upper approximation operators on $X$, respectively.

Obviously,

$\mathrm{SE}-\mathbf{L A O}(\mathbf{X}), \operatorname{RE}-\mathbf{L A O}(\mathbf{X}), \mathrm{TR}-\mathbf{L A O}(\mathbf{X}) \subseteq \mathbf{L A O}(X)$,

$\mathrm{SE}-\mathbf{U A O}(\mathbf{X}), \mathrm{RE}-\mathbf{U A O}(\mathbf{X}), \mathrm{TR}-\mathbf{U A O}(\mathbf{X}) \subseteq \mathbf{U A O}(\mathbf{X})$.

\section{Theorem 1}

(1) Define a relation $\subseteq$ on $\mathbf{L}-\mathbf{F G N S}(\mathbf{X})$ as follows: $N_{1} \subseteq N_{2}$ if and only if $N_{1}(x)(C) \leq N_{2}(x)(C)$ for any $x \in X$ and $C \in L^{X}$. Then, $(\mathbf{L}-\mathbf{F G N S}(\mathbf{X}), \subseteq)$ is a poset.
(2) Define a relation $\leq$ on $\mathbf{L A O}(\mathbf{X})$ as follows: $N_{1} \leq N_{2}$ if and only if $N_{1}(B)(x) \leq N_{2}(B)(x)$ for any $x \overline{\epsilon X}$ and $B \in L^{X}$. Then, $(\mathbf{L A O}(\overline{\mathbf{X}}), \leq)$ is a poset.

(3) Define a relation $\leq$ on $\mathbf{U A O}(\mathbf{X})$ as follows: $\overline{N_{1}} \leq \overline{N_{2}}$ if and only if $\overline{N_{2}}(B)(x) \leq \overline{N_{1}}(B)(x)$ for any $x \in X$ and $B \in L^{X}$. Then, $(\mathbf{U A O}(\mathbf{X}), \leq)$ is a poset.

Proof. The proof is straightforward and is omitted.

Proposition 2. Let $\Lambda$ be an index set; $\forall\left\{N_{i}\right\}_{i \in \Lambda} \subseteq(\mathbf{L}-\mathbf{F G N S}(\mathbf{X}), \subseteq)$, we can define union of $N_{i}$ as follows:

$$
\left(\bigcup_{i \in \Lambda} N_{i}\right)(x)(C)=\vee_{i \in \Lambda} N_{i}(x)(C),
$$

for any $x \in X$ and $C \in L^{X}$; then, $\cup_{i \in \Lambda} N_{i} \in \mathbf{L}-$ FGNS (X). Furthermore,

(1) If $\left\{N_{i}\right\}_{i \in \Lambda} \subseteq S E-\operatorname{LFGNS}(\mathbf{X})$, then $\cup_{i \in \Lambda} N_{i} \in S E-$ LFGNS (X).

(2) If $\left\{N_{i}\right\}_{i \in \Lambda} \subseteq R E-\operatorname{LFGNS}(\mathbf{X})$, then $\cup_{i \in \Lambda} N_{i} \in$ $R E$ - LFGNS $(\mathrm{X})$.

(3) If $\left\{N_{i}\right\}_{i \in \Lambda} \subseteq T R-\operatorname{LFGNS}(\mathbf{X})$, then $\cup_{i \in \Lambda} N_{i}$ $\in T R$ - LFGNS $(\mathbf{X})$.

Proof. The proof of (1) and (2) are straightforward and are omitted. (3) Note that TR - LFGNS (X) $\subseteq \mathbf{L}-$ FGNS (X). So, if $\left\{N_{i}\right\}_{i \in \Lambda} \subseteq$ TR - LFGNS $(\mathbf{X})$, then there must be $\cup_{i \in \Lambda} N_{i} \in \mathbf{L}-$ FGNS $(\mathbf{X})$.

$$
\begin{aligned}
& \forall x \in X, C \in L^{X} \\
& \left(\cup_{i \in \Lambda} N_{i}\right)(x)(C)=\vee_{i \in \Lambda} N_{i}(x)(C) \\
& \leq \underset{i \in \Lambda}{\vee} \underset{V \in L^{X}}{\vee}\left[N_{i}(x)(V) * \underset{y \in X}{\wedge}\left(V(y) \longrightarrow \underset{V_{y} \in L^{X}}{\vee}\left(N_{i}(y)\left(V_{y}\right) * S\left(V_{y}, C\right)\right)\right)\right] \\
& =\underset{V \in L^{X}}{\vee} \underset{i \in \Lambda}{\vee}\left[N_{i}(x)(V) * \underset{y \in X}{\wedge}\left(V(y) \longrightarrow \underset{V_{y} \in L^{X}}{\vee}\left(N_{i}(y)\left(V_{y}\right) * S\left(V_{y}, C\right)\right)\right)\right] \\
& \leq \underset{V \in L^{X}}{\vee} \underset{(i, j) \in \Lambda \times \Lambda}{\vee}\left[N_{i}(x)(V) * \underset{y \in X}{\wedge}\left(V(y) \longrightarrow \underset{V_{y} \in L^{X}}{\vee}\left(N_{i}(y)\left(V_{y}\right) * S\left(V_{y}, C\right)\right)\right)\right] \\
& =\underset{V \in L^{X}}{\vee}\left[\underset{i \in \Lambda}{\vee} N_{i}(x)(V) * \underset{j \in \Lambda}{\vee}\left(\underset{y \in X}{\wedge}\left(V(y) \longrightarrow \underset{V_{y} \in L^{X}}{\vee}\left(N_{i}(y)\left(V_{y}\right) * S\left(V_{y}, C\right)\right)\right)\right]\right. \\
& \leq \underset{V \in L^{X}}{\vee}\left[\underset{i \in \Lambda}{\vee} N_{i}(x)(V) * \underset{y \in X}{\wedge}\left(V(y) \longrightarrow \underset{i \in \Lambda}{\vee} \underset{V_{y} \in L^{X}}{\vee}\left(N_{i}(y)\left(V_{y}\right) * S\left(V_{y}, C\right)\right)\right)\right] \\
& =\underset{V \in L^{X}}{\vee}\left[\underset{i \in \Lambda}{\vee} N_{i}(x)(V) * \underset{y \in X}{\wedge}\left(V(y) \longrightarrow \underset{V_{y} \in L^{X}}{\vee}\left(\underset{i \in \Lambda}{\vee} N_{i}(y)\left(V_{y}\right) * S\left(V_{y}, C\right)\right)\right)\right] \\
& =\underset{V \in L^{X}}{\vee}\left[\left(\cup_{i \in \Lambda}^{\cup} N_{i}\right)(x)(V) * \underset{y \in X}{\wedge}\left(V(y) \longrightarrow \underset{V_{y} \in L^{X}}{\vee}\left(\left(\cup_{i \in \Lambda}^{\cup} N_{i}\right)(y)\left(V_{y}\right) * S\left(V_{y}, C\right)\right)\right)\right] .
\end{aligned}
$$


Thus, $\cup_{i \in \Lambda} N_{i} \in \mathbb{C}$. Therefore, $\cup_{i \in \Lambda} N_{i} \in \mathbf{L}-\operatorname{FGNS}(\mathbf{X})$ $\cap \mathbb{C}=$ TR - LFGNS $(\mathbf{X})$.

By Proposition 2, we obtain the following theorem.

Theorem 2. (L - FGNS $(\mathrm{X}), \subseteq, \cup), \quad(S E-\operatorname{LFGNS}(\mathbf{X}), \subseteq$, $\cup),(R E-\operatorname{LFGNS}(\mathbf{X}), \subseteq, \cup)$, and $(T R-\operatorname{LFGNS}(\mathbf{X}), \subseteq, \cup)$ are all join-complete lattices.

\section{Theorem 3}

(1) $\forall\left\{\underline{N}_{i}\right\}_{i \in \Lambda} \subseteq(\mathbf{L A O}(\mathbf{X}), \leq)$ and $\Lambda$ be an index set, we can define union of $N_{i}$ as follows:

$$
\underset{i \in \Lambda}{\vee} N_{i}=\underline{\underline{i \in \Lambda}} N_{i}
$$

Then, $\vee_{i \in \Lambda} \underline{N_{i}}=\underline{\cup_{i \in \Lambda} N_{i}}$ is the supremum of $\left\{\underline{N_{i}}\right\}_{i \in \Lambda}$.

(2) $\forall\left\{\bar{N}_{i}\right\}_{i \in \Lambda} \subseteq(\mathbf{U A O}(\mathbf{X}), \leq)$ and $\Lambda$ be an index set, we can define union of $\overline{N_{i}}$ as follows:

$$
\bigvee_{i \in \Lambda} \overline{N_{i}}=\overline{\bigcup_{i \in \Lambda} N_{i}}
$$

Then, $\vee_{i \in \Lambda} \overline{N_{i}}=\overline{\cup_{i \in \Lambda} N_{i}}$ is the supremum of $\left\{\overline{N_{i}}\right\}_{i \in \Lambda}$.

Proof

Step 1. We first prove that the following equation holds: for any $x \in X$ and $B \in L^{X}$,

$$
\begin{aligned}
& \underset{i \in \Lambda}{\cup} N_{i}(B)(x)=\bigvee_{i \in \Lambda} \underline{N_{i}}(B)(x), \\
& \overline{\bigcup_{i \in \Lambda} N_{i}}(B)(x)=\bigwedge_{i \in \Lambda} \overline{N_{i}}(B)(x) .
\end{aligned}
$$

In fact,

$$
\begin{aligned}
& \cup_{i \in \Lambda} N_{i}(B)(x)=\underset{C \in L^{x}}{\vee}\left[\left(\cup_{i \in \Lambda} N_{i}\right)(x)(C) * S(C, B)\right] \\
& =\underset{C \in L^{X}}{\vee}\left[\underset{i \in \Lambda}{\vee} N_{i}(x)(C) * S(C, B)\right] \\
& =\underset{C \in L^{X}}{\vee} \underset{i \in \Lambda}{\vee}\left(N_{i}(x)(C) * S(C, B)\right) \\
& =\underset{i \in \Lambda}{\vee} \underset{C \in L^{X}}{\vee}\left(N_{i}(x)(C) * S(C, B)\right) \\
& =\underset{i \in \Lambda}{\vee} \frac{N_{i}}{}(B)(x) \text {, } \\
& \overline{\cup_{i \in \Lambda} N_{i}}(B)(x)=\wedge_{C \in L^{X}}\left[\left(\cup_{i \in \Lambda} N_{i}\right)(x)(C) \longrightarrow I(C, B)\right] \\
& =\wedge \wedge_{C \in L^{X}}\left[\underset{i \in \Lambda}{\vee} N_{i}(x)(C) \longrightarrow I(C, B)\right] \\
& =\wedge \wedge_{C \in L^{X}} \wedge\left(N_{i \in \Lambda}(x)(C) \longrightarrow I(C, B)\right) \\
& =\wedge_{i \in \Lambda} \wedge_{C \in L^{X}}\left(N_{i}(x)(C) \longrightarrow I(C, B)\right) \\
& =\wedge \bar{N}_{i \in \Lambda}(B)(x) \text {. }
\end{aligned}
$$

\section{Step 2}

(1) Let $N=\cup_{i \in \Lambda} N_{i}$; then, $N_{i} \subseteq N$ for each $i \in \Lambda$. By Step 1, we have $N_{i} \leq \underline{N}$. If $N^{*}$ is another $L$-fuzzy generalized neighborhood system operator on $X$ such that $N_{i} \leq \underline{N^{*}}$ for each $i \in \Lambda$, then $\underline{N_{i}}(B)(x) \leq \underline{\overline{N^{*}}}(B) \overline{(x)}$ for any $x, B \in L^{X}$. Hence,

$\underline{N}(B)(x)=\underline{\underline{i \in \Lambda}} N_{i}(B)(x)=\underset{i \in \Lambda}{\vee} \underline{N_{i}}(B)(x) \leq \underline{N^{*}}(B)(x)$.

Thus, $\underline{N} \leq \underline{N^{*}}$. So, $\vee_{i \in \Lambda} \underline{N_{i}}=\underline{N}=\underline{\cup_{i \in \Lambda} N_{i}}$ is the supremum of $\left\{N_{i}\right\}_{i \in \Lambda}$.

(2) Let $N=\cup_{i \in \Lambda} N_{i}$; then, $N_{i} \subseteq N$ for each $i \in \Lambda$. By Step 1 , we have $\overline{N_{i}} \leq \bar{N}$. If $N^{*}$ is another $L$-fuzzy generalized neighborhood system operator on $X$ such that $\overline{N_{i}} \leq \overline{N^{*}}$ for each $i \in \Lambda$, then $\overline{N^{*}}(B)(x) \leq \overline{N_{i}}(B)(x)$ for any $x, B \in L^{X}$. Hence, 
$\overline{N^{*}}(B)(x) \leq \bigwedge_{i \in \Lambda} \overline{N_{i}}(B)(x)=\overline{\bigcup_{i \in \Lambda} N_{i}}(B)(x)=\bar{N}(B)(x)$.

Thus, $\bar{N} \leq \overline{N^{*}}$. So, $\vee_{i \in \Lambda} \overline{N_{i}}=\bar{N}=\overline{U_{i \in \Lambda} N_{i}}$ is the supremum of $\left\{\overline{N_{i}}\right\}_{i \in \Lambda}$.

By Proposition 2 and Theorems 2 and 3, we can obtain Theorem 4 as follows.

Theorem 4. The posets $(\mathbf{L A O}(\mathbf{X}), \leq, \mathrm{V}),(\mathbf{U A O}(\mathbf{X}), \leq, \mathrm{V})$, $(S E-\mathbf{L A O}(\mathbf{X}), \leq, \vee), \quad(S E-\mathbf{U A O}(\mathbf{X}), \leq, \vee), \quad(R E-\mathbf{L A O}$ $(\mathbf{X}), \leq, \mathrm{V}), \quad(R E-\mathbf{U A O}(\mathbf{X}), \leq, \mathrm{V}), \quad(T R-\mathbf{L A O}(\mathbf{X}), \leq, \mathrm{V})$ and $(T R-\mathbf{U A O}(\mathbf{X}), \leq, V)$ are all join-complete lattices.

Furthermore, we have the following theorem.

\section{Theorem 5}

(1) If L satisfies the double negative law, then the joincomplete lattices $(\mathbf{L A O}(\mathbf{X}), \leq, \mathrm{V})$ and ( $\mathbf{U A O}(\mathbf{X}), \leq, V)$ are order isomorphic.

(2) If L satisfies the double negative law, when L-fuzzy generalized neighborhood system is serial, reflexive, and transitive, there still exists an order isomorphic mapping between upper and lower approximation operators, respectively.

Proof. We only show (1).

Step 1. Define a mapping $f: \mathbf{L A O}(\mathbf{X}) \longrightarrow \mathbf{U A O}(\mathbf{X})$ as follows: for any $A \in L^{X}$ and for any $x \in X$, $f(\underline{N})(A)(x)=\neg \underline{N}(\neg A)(x)$ for each $\underline{N} \in \mathbf{L A O}(\mathbf{X})$; then, $f(\underline{N}) \in \overline{\mathbf{U A O}}(\mathbf{X})$. Define a mapping g: $\mathbf{U A O}(\mathbf{X}) \longrightarrow \operatorname{LAO}(\mathbf{X})$ as follows: for any $A \in L^{X}$ and for any $x \in X, g(\bar{N})(A)(x)=\neg \bar{N}(\neg A)(x)$ for each $\bar{N} \in \mathbf{U A O}(\mathbf{X})$; then, $g(\bar{N}) \in \mathbf{L A O}(\mathbf{X})$.

In fact, by Theorem 3.9 of [50], if $L$ satisfies the double negative law, then we have $f(\underline{N})(A)(x)=\neg \underline{N}(\neg A)$ $(x)=\bar{N}(A)(x)$ for any $A \in L^{X}$ and for any $x \in X$. So, $f(\underline{\underline{N}})=\bar{N} \in \mathbf{U A O}(\mathbf{X})$. Similarly, we can prove that $g(\bar{N}) \in \mathbf{L A O}(\mathbf{X})$.

Step 2. $f \circ g=\mathrm{id}_{\mathbf{U A O}(\mathbf{X})}$ and $g \circ f=\operatorname{id}_{\mathbf{L A O}(\mathbf{X})}$, where $\mathrm{id}_{\mathrm{UAO}(\mathbf{X})}$ (resp., $\mathrm{id}_{\mathbf{L A O}(\mathbf{X})}$ ) is the identity mapping on $\mathbf{U A O}(\mathbf{X})$ (resp., $\mathbf{L A O}(\mathbf{X}))$.

In fact, $\forall \bar{N} \in \mathbf{U A O}(\mathbf{X})$, for any $A \in L^{X}$ and for any $x \in X$, by Step 1 , we have

$$
(f \circ g)(\bar{N})(A)(x)=f(\underline{N})(A)(x)=\bar{N}(A)(x)=\operatorname{id}_{\mathbf{U A O}(\mathbf{X})}(\bar{N})(A)(x) .
$$

Hence, $f \circ g=\mathrm{id}_{\mathbf{U A O}(\mathbf{X})}$.

$\forall \underline{N} \in \mathbf{L A O}(\mathbf{X})$, for any $A \in L^{X}$ and for any $x \in X$, by Step 1, we have

$$
(g \circ f)(\underline{N})(A)(x)=g(\bar{N})(A)(x)=\underline{N}(A)(x)=\operatorname{id}_{\mathbf{L A O}(\mathbf{X})}(\underline{N})(A)(x) .
$$

Hence, $g \circ f=\mathrm{id}_{\mathbf{L A O}(\mathbf{X})}$.

Step 3. The mappings $f: \mathbf{L A O}(\mathbf{X}) \longrightarrow \mathbf{U A O}(\mathbf{X})$ and $f^{-1}=g: \mathbf{U A O}(\mathbf{X}) \longrightarrow \mathbf{L A O}(\mathbf{X})$ are order-preserving mappings.

In fact, let $N_{1}, N_{2} \in \mathbf{L A O}(\mathbf{X})$ and $N_{1} \leq N_{2}$; by Step 1

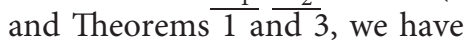

$$
\overline{N_{2}}=f\left(\underline{N_{2}}\right)=f\left(\underline{N_{1} \cup N_{2}}\right)=\overline{N_{1} \cup N_{2}}=\overline{N_{1}} \vee \overline{N_{2}} .
$$
have

So, for any $A \in L^{X}$ and for any $x \in X$, by Theorem 3, we

$$
\overline{N_{2}}(A)(x)=\left(\overline{N_{1}} \vee \overline{N_{2}}\right)(A)(x)=\overline{N_{1}}(A)(x) \wedge \overline{N_{2}}(A)(x) .
$$
have

It implies that $\overline{N_{2}}(A)(x) \leq \overline{N_{1}}(A)(x)$. By Theorem 1 , we

$$
f\left(\underline{N_{1}}\right)=\overline{N_{1}} \leq \overline{N_{2}}=f\left(\underline{N_{2}}\right) .
$$

Hence, the mapping $f: \mathbf{L A O}(\mathbf{X}) \longrightarrow \mathbf{U A O}(\mathbf{X})$ is an order-preserving mapping. Similarly, we can prove that $f^{-1}=g$ : $\mathbf{U A O}(\mathbf{X}) \longrightarrow \mathbf{L A O}(\mathbf{X})$ is also an order-preserving mapping.

From Steps 1 to 3 , we know that the join-complete lattices $(\mathbf{L A O}(\mathbf{X}), \leq, \vee)$ and $(\mathbf{U A O}(\mathbf{X}), \leq, \vee)$ are order isomorphic. 


\section{The Lattice Structures of Approximation Operators Based on $L$-Fuzzy Relations}

We know that approximation operators based on $L$-fuzzy relations $[4,37]$ can be regarded as special approximation operators based on $L$-fuzzy generalized neighborhood systems (see Lemma 2.7, also see [50]). So, in this section, we will study the lattice structures of approximation operators based on $L$-fuzzy relations.

Theorem 6. Let $\Lambda$ be an index set; for any $x \in X$ and $A \in L^{X}$,

$$
\begin{aligned}
& \text { (1) } \underset{i \in \Lambda}{\vee} R_{i}(A)(x)=\wedge \wedge_{i \in \Lambda} \underline{R_{i}}(A)(x) \text {, } \\
& \underset{i \in \Lambda}{\wedge} R_{i}(A)(x) \geq \underset{i \in \Lambda}{\vee} \underline{R_{i}}(A)(x),
\end{aligned}
$$

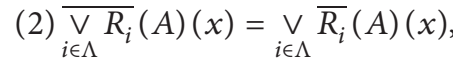

$$
\begin{aligned}
& \overline{\bigwedge_{i \in \Lambda} R_{i}}(A)(x) \leq \wedge \bar{R}_{i \in \Lambda}(A)(x) .
\end{aligned}
$$

Proof. For any $x \in X$ and $A \in L^{X}$,

$$
\begin{aligned}
& \text { (1) } \underset{i \in \Lambda}{\vee} R_{i}(A)(x)=\wedge_{y \in X}\left[\left(\vee_{i \in \Lambda}^{\vee} R_{i}\right)(x, y) \longrightarrow A(y)\right] \\
& =\wedge \wedge_{y \in X}\left[\vee_{i \in \Lambda} R_{i}(x, y) \longrightarrow A(y)\right] \\
& =\wedge \wedge_{y \in X} \wedge\left[R_{i \in \Lambda}(x, y) \longrightarrow A(y)\right] \\
& =\wedge \wedge_{i \in \Lambda} \wedge\left[R_{y \in X}(x, y) \longrightarrow A(y)\right] \\
& \underset{i \in \Lambda}{\wedge} R_{i}(A)(x)=\wedge_{y \in X}\left[\left(\wedge_{i \in \Lambda} R_{i}\right)(x, y) \longrightarrow A(y)\right] \\
& =\wedge \wedge_{y \in X}\left[{ }_{i \in \Lambda} R_{i}(x, y) \longrightarrow A(y)\right] \\
& \geq \underset{i \in \Lambda}{\vee} \wedge_{y \in X}\left(R_{i}(x, y) \longrightarrow A(y)\right) \\
& =\underset{i \in \Lambda}{\vee} \underline{R_{i}}(A)(x), \\
& \text { (2) } \underset{i \in \Lambda}{\vee} R_{i}(A)(x)=\underset{y \in X}{\vee}\left[\left(\vee_{i \in \Lambda} R_{i}\right)(x, y) * A(y)\right] \\
& =\underset{y \in X}{\vee}\left[\vee_{i \in \Lambda} R_{i}(x, y) * A(y)\right] \\
& =\underset{i \in \Lambda}{\vee} \underset{y \in X}{\vee}\left(R_{i}(x, y) * A(y)\right) \\
& \widehat{{ }_{i \in \Lambda} R_{i}}(A)(x)=\underset{y \in X}{\vee}\left[\left(\wedge_{i \in \Lambda} R_{i}\right)(x, y) * A(y)\right] \\
& =\underset{y \in X}{\vee}\left[\hat{i \in \Lambda} R_{i}(x, y) * A(y)\right] \\
& \leq \underset{i \in \Lambda}{\vee} \underset{y \in X}{\vee}\left(R_{i}(x, y) * A(y)\right) \\
& =\wedge_{i \in \Lambda} \overline{R_{i}}(A)(x) .
\end{aligned}
$$

Let $\quad \operatorname{RLAO}(\mathbf{X})=\left\{\underline{R}: L^{X} \longrightarrow L^{X} \mid R \in L^{X \times X}\right\} \quad$ and $\operatorname{RUAO}(\mathbf{X})=\left\{\bar{R}: L^{X} \longrightarrow L^{X} \mid R \in L^{X \times X}\right\}$ be the family of all lower and upper approximation operators based on $L$-fuzzy relations, respectively.

Define a relation $\leq$ on $\mathbf{R L A O}(\mathbf{X})$ as follows: $R_{1} \leq R_{2}$ if and only if $R_{2}(B)(x) \leq R_{1}(B)(x)$ for any $x \bar{\epsilon} X$ and $B \in L^{X}$. Then, $(\mathbf{R L A O}(\mathbf{X}), \leq)$ is a poset, and $0_{X \times X}$ is its bottom element.

Define a relation $\leq$ on $\mathbf{R U A O}(\mathbf{X})$ as follows: $\overline{R_{1}} \leq \overline{R_{2}}$ if and only if $\overline{R_{1}}(B)(x) \leq \overline{R_{2}}(B)(x)$ for any $x \in X$ and $B \in L^{X}$. Then, $(\operatorname{RUAO}(\mathbf{X}), \leq)$ is a poset, and $\overline{0_{X \times X}}$ is its bottom element.

Theorem 7. Let $\Lambda$ be an index set.

(1) $\forall\left\{\underline{R}_{i}\right\}_{i \in \Lambda} \subseteq \mathbf{R L A O}(\mathbf{X})$, we can define union and intersection of $R_{i}$ as follows:

$$
\begin{aligned}
& \underset{i \in \Lambda \underline{R_{i}}}{\vee}=\underset{i \in \Lambda}{\vee} R_{i}, \\
& \underset{i \in \Lambda \underline{R_{i}}}{\wedge} \underline{\left\langle\hat{\Lambda \in \Lambda} R_{i}\right\rangle},
\end{aligned}
$$

where

$$
\begin{aligned}
\left\langle\wedge \wedge_{i \in \Lambda} R_{i}\right\rangle & =\vee\left\{R \in L^{X \times X} \mid \forall A \in L^{X}, \forall x \in X, \underline{R}(A)(x)\right. \\
& \left.\geq \underset{i \in \Lambda}{\vee} \underline{R_{i}}(A)(x)\right\} .
\end{aligned}
$$

Then, $\vee_{i \in \Lambda} R_{i}$ and $\wedge_{i \in \Lambda} R_{i}$ are supremum and infimum of $\left\{\underline{R_{i}}\right\}_{i \in \Lambda}$ respectively.

(2) $\forall\left\{\overline{R_{i}}\right\}_{i \in \Lambda} \subseteq \mathbf{R U A O}(\mathbf{X})$, we can define union and intersection of $\overline{R_{i}}$ as follows:

$$
\vee_{i \in \Lambda} \overline{R_{i}}=\overline{\vee_{i \in \Lambda} R_{i}} \cdot \underset{i \in \Lambda}{\wedge} \overline{R_{i}}=\overline{\left[\wedge \wedge_{i \in \Lambda} R_{i}\right]}
$$

where

$$
\left[\wedge_{i \in \Lambda} R_{i}\right]=\vee\left\{R \in L^{X \times X} \mid \forall A \in L^{X}, \forall x \in X, \bar{R}(A)(x) \leq{ }_{i \in \Lambda} \overline{R_{i}}(A)(x)\right\} .
$$

Then, $\vee_{i \in \Lambda} \overline{R_{i}}$ and $\wedge_{i \in \Lambda} \overline{R_{i}}$ are supremum and infimum of $\left\{\bar{R}_{i}\right\}_{i \in \Lambda}$, respectively.

Proof

(1) We only show that $\wedge_{i \in \Lambda} R_{i}$ is the infimum of $\left\{R_{i}\right\}_{i \in \Lambda}$. Note that $0_{X \times X}(A)(x)=1$; for any $A \in L^{X}$ and for any $x \in X$, we have

$$
0_{X \times X} \in\left\{R \in L^{X \times X} \mid \forall A \in L^{X}, \forall x \in X, \underline{R}(A)(x) \geq \underset{i \in \Lambda}{\vee} \underline{R_{i}}(A)(x)\right\} .
$$

So, $0_{X \times X} \leq\left\langle\wedge_{i \in \Lambda} R_{i}\right\rangle \in L^{X \times X}$. Hence, $0_{X \times X} \leq\left\langle\wedge_{i \in \Lambda}\right.$ $\left.R_{i}\right\rangle$. Furthermore, for any $x \in X$ and $B \in L^{X}$, by Theorem 6, we have 
$\left\langle\underline{i \in \Lambda} R_{i}\right\rangle(B)(x) \geq \underset{i \in \Lambda \underline{R_{i}}}{\vee}(B)(x) \geq \underline{R_{i}}(B)(x)$,

for each $i \in \Lambda$. It implies that $\underline{0_{X \times X}} \leq \underline{\left\langle\Lambda_{i \in \Lambda} R_{i}\right\rangle} \leq \underline{R_{i}}$ for each $i \in \Lambda$.

If $R^{*}$ is another $L$-fuzzy relation on $X$ such that $\underline{R^{*}} \leq R_{i}$ for each $i \in \Lambda$, then for any $x \in X$ and $\overline{A \in L^{X}}$, we have $R_{i}(A)(x) \leq \underline{R^{*}}(A)(x)$. So, $\vee_{i \in \Lambda} R_{i}(A)(x) \leq \underline{R^{*}}(A)(x)$. By the construction of $\left\langle\wedge_{i \in \Lambda} R_{i}\right\rangle$, we can easily obtain $\left\langle\wedge_{i \in \Lambda} R_{i}\right\rangle \geq R^{*}$. Thus, $\left\langle\wedge_{i \in \Lambda} R_{i}\right\rangle \geq \underline{R^{*}}$. Hence, $\wedge_{i \in \Lambda} \underline{R_{i}}=\underline{\left\langle\Lambda_{i \in \Lambda} R_{i}\right\rangle}$ is the infimum of $\left\{R_{i}\right\}_{i \in \Lambda}$.

(2) We only show that $\Lambda_{i \in \Lambda} \overline{R_{i}}$ is the infimum of $\left\{\overline{R_{i}}\right\}_{i \in \Lambda}$.

Note that $\overline{0_{X \times X}}(A)(x)=0$; for any $A \in L^{X}$ and for any $x \in X$, we have

$$
0_{X \times X} \in\left\{R \in L^{X \times X} \mid \forall A \in L^{X}, \forall x \in X, \bar{R}(A)(x) \leq \underset{i \in \Lambda}{\wedge} \overline{R_{i}}(A)(x)\right\} .
$$

So, $0_{X \times X} \leq\left[\wedge_{i \in \Lambda} R_{i}\right] \in L^{X \times X}$. Hence, $\overline{0_{X \times X}} \leq \overline{\left[\wedge_{i \in \Lambda} R_{i}\right]}$. Furthermore, for any $x \in X$ and $B \in L^{X}$, by Theorem 6 , we have

$$
\overline{\left[\hat{i \in \Lambda}_{i} R_{i}\right]}(B)(x) \leq \wedge_{i \in \Lambda} \underline{R_{i}}(B)(x) \leq \overline{R_{i}}(B)(x)
$$

for each $i \in \Lambda$. It implies that $\overline{0_{X \times X}} \leq \overline{\left[\wedge_{i \in \Lambda} R_{i}\right]} \leq \overline{R_{i}}$ for each $i \in \Lambda$.

If $R^{*}$ is another $L$-fuzzy relation on $X$ such that $\overline{R^{*}} \leq \overline{R_{i}}$ for each $i \in \Lambda$, then for any $x \in X$ and $A \in L^{X}$, we have $\overline{R_{i}}(A)(x) \geq \overline{R^{*}}(A)(x)$. So, $\wedge_{i \in \Lambda} \overline{R_{i}}(A)(x) \geq \overline{R^{*}}(A)(x)$. By the construction of $\left[\wedge_{i \in \Lambda} R_{i}\right]$, we can easily obtain $\left[\wedge_{i \in \Lambda} R_{i}\right] \geq R^{*}$. Thus, $\overline{\left[\wedge_{i \in \Lambda} R_{i}\right]} \geq \overline{R^{*}}$. Hence, $\wedge_{i \in \Lambda} \overline{R_{i}}=\overline{\left[\wedge_{i \in \Lambda} R_{i}\right]}$ is the infimum of $\left\{\overline{R_{i}}\right\}_{i \in \Lambda}$.

By Theorem 7 , we know that both $(\operatorname{RLAO}(\mathbf{X}), \leq, \vee, \wedge)$ and $(\mathbf{R U A O}(\mathbf{X}), \leq, \vee, \wedge)$ are complete lattices. Furthermore, we have the following conclusion.

Theorem 8. If $L$ satisfies the double negative law, then both $(\mathbf{R L A O}(\mathbf{X}), \leq, \vee, \wedge)$ and $(\mathbf{R U A O}(\mathbf{X}), \leq, \vee, \wedge)$ are complete lattice isomorphism.

Proof. Note that both $(\operatorname{RLAO}(\mathbf{X}), \leq, \vee, \wedge)$ and $(\mathbf{R U A O}(\mathbf{X}), \leq, \vee, \wedge)$ are complete lattices. So, we only need to prove the following steps.

Step 1. Define a mapping F: $\mathbf{R L A O}(\mathbf{X}) \longrightarrow \operatorname{RUAO}(\mathbf{X})$ as follows: for any $A \in L^{X}$ and for any $x \in X$, $F(\underline{R})(A)(x)=\neg \underline{R}(\neg A)(x)$ for each $\underline{R} \in \mathbf{R L A O}(\mathbf{X})$; then, $F(\underline{R}) \in \mathbf{R U A O}(\mathbf{X})$.

In fact, $\forall \underline{R} \in \mathbf{R L A O}(\mathbf{X})$, for any $A \in L^{X}$ and for any $x \in X$, we have

$$
\begin{aligned}
F(\underline{R})(A)(x) & =\neg \underline{R}(\neg A)(x) \\
& =\neg \wedge_{y \in X}(R(x, y) \longrightarrow \neg A(y)) \\
& =\vee_{y \in X} \neg(R(x, y) \longrightarrow \neg A(y)) \\
& =\vee_{y \in X}(R(x, y) * A(y))=\bar{R}(A)(x) .
\end{aligned}
$$

Hence, $F(\underline{R})=\bar{R} \in \mathbf{R U A O}(\mathbf{X})$.

Step 2. Define a mapping $G$ : $\mathbf{R U A O}(\mathbf{X}) \longrightarrow \operatorname{RLAO}(\mathbf{X})$ as follows: for any $A \in L^{X}$ and for any $x \in X$, $G(\bar{R})(A)(x)=\neg \bar{R}(\neg A)(x)$ for each $\bar{R} \in \mathbf{R U A O}(\mathbf{X})$; then, $G(\bar{R}) \in \mathbf{R L A O}(\mathbf{X})$.

In fact, $\forall \bar{R} \in \mathbf{R U A O}(\mathbf{X})$, for any $A \in L^{X}$ and for any $x \in X$, we have

$$
\begin{aligned}
G(\bar{R})(A)(x) & =\neg \bar{R}(\neg A)(x) \\
& =\neg \bigvee_{y \in X}(R(x, y) * \neg A(y)) \\
& =\bigwedge_{y \in X} \neg(R(x, y) * \neg A(y)) \\
& =\bigwedge_{y \in X}(R(x, y) \longrightarrow A(y)) \\
& =\underline{R}(A)(x) .
\end{aligned}
$$

Hence, $G(\bar{R})=\underline{R} \in \mathbf{R L A O}(\mathbf{X})$.

Step 3. $F \circ G=\mathrm{id}_{\mathrm{RUAO}(\mathrm{X})}$ and $G \circ F=\mathrm{id}_{\mathrm{RLAO}(\mathbf{X})}$, where $\mathrm{id}_{\mathrm{RUAO}(\mathbf{X})}$ (resp., $\operatorname{id}_{\mathrm{RLAO}(\mathbf{X})}$ ) is the identity mapping on RUAO (X) (resp., $\operatorname{RLAO}(\mathbf{X})$ ).

In fact, $\forall \bar{R} \in \mathbf{R U A O}(\mathbf{X})$, for any $A \in L^{X}$ and for any $x \in X$, we have

$$
\begin{aligned}
& (F \circ G)(\bar{R})(A)(x)=F(\underline{R})(A)(x)=\bar{R}(A)(x) \\
& =\operatorname{id}_{\mathbf{R U A O}(\mathbf{X})}(\bar{R})(A)(x) .
\end{aligned}
$$

Hence, $F \circ G=\operatorname{id}_{\operatorname{RUAO}(\mathbf{X})} \cdot \forall \underline{R} \in \mathbf{R L A O}(\mathbf{X})$, for any $A \in L^{X}$ and for any $x \in X$,

$$
\begin{aligned}
& (G \circ F)(\underline{R})(A)(x)=G(\bar{R})(A)(x)=\underline{R}(A)(x) \\
& =\operatorname{id}_{\operatorname{RLAO}(\mathbf{X})}(\underline{R})(A)(x) .
\end{aligned}
$$

Hence, $G \circ F=\operatorname{id}_{\operatorname{RLAO}(\mathbf{X})}$.

Step 4. The mapping $F: \operatorname{RLAO}(\mathbf{X}) \longrightarrow \operatorname{RUAO}(\mathbf{X})$ preserves arbitrary union and arbitrary intersection.

In fact, $\forall\left\{\underline{R}_{i}\right\}_{i \in \Lambda} \subseteq \mathbf{R L A O}(\mathbf{X})$ and $\Lambda$ be an index set. On the one hand, by Theorem 7 and Step 1, we have

$$
F\left(\vee_{i \in \Lambda} \underline{R_{i}}\right)=F\left(\underset{\underline{i \in \Lambda}}{\vee} R_{i}\right)=\overline{\vee_{i \in \Lambda} R_{i}}=\vee_{i \in \Lambda} \overline{R_{i}}=\vee_{i \in \Lambda} F\left(\underline{R_{i}}\right) .
$$

So, the mapping $F: \mathbf{R L A O}(\mathbf{X}) \longrightarrow \operatorname{RUAO}(\mathbf{X})$ preserves arbitrary union.

On the other hand, 


$$
\begin{aligned}
& R_{0} \in\left\{R \in L^{X \times X} \mid \forall A \in L^{X}, \forall x \in X, \bar{R}(A)(x) \leq \wedge \wedge_{i \in \Lambda} \overline{R_{i}}(A)(x)\right\} \\
& \Longleftrightarrow \forall A \in L^{X}, \forall x \in X, \overline{R_{0}}(A)(x) \leq \wedge \underset{i \in \Lambda}{\wedge} \overline{R_{i}}(A)(x) \\
& \Longleftrightarrow \forall A \in L^{X}, \forall x \in X, \overline{R_{0}}(\neg A)(x) \leq \wedge \wedge_{i \in \Lambda} \overline{R_{i}}(\neg A)(x) \\
& \Longleftrightarrow \forall A \in L^{X}, \forall x \in X, \neg \overline{R_{0}}(\neg A)(x) \geq \neg \wedge \wedge_{i \in \Lambda} \overline{R_{i}}(\neg A)(x) \Longleftrightarrow \forall A \in L^{X}, \quad \forall x \in X, \\
& \underline{R_{0}}(A)(x)=\neg \overline{R_{0}}(\neg A)(x) \geq \neg \wedge_{i \in \Lambda} \overline{R_{i}}(\neg A)(x)=\underset{i \in \Lambda}{\vee} \neg \overline{R_{i}}(\neg A)(x)=\underset{i \in \Lambda}{\vee} \underline{R_{i}}(A)(x) \\
& \Longleftrightarrow \forall A \in L^{X}, \forall x \in X, \underline{R_{0}}(A)(x) \geq \underset{i \in \Lambda}{\vee} \underline{R_{i}}(A)(x) \\
& \Longleftrightarrow R_{0} \in\left\{R \in L^{X \times X} \mid \forall A \in L^{X}, \forall x \in X, \underline{R}(A)(x) \geq \underset{i \in \Lambda}{\vee} \underline{R_{i}}(A)(x)\right\} .
\end{aligned}
$$

It implies that $\left\langle\wedge_{i \in \Lambda} R_{i}\right\rangle=\left[\wedge_{i \in \Lambda} R_{i}\right]$. Hence, by Theorem 7 and Step 1, we have

$$
\begin{aligned}
& \left.F\left(\hat{i \in \Lambda}_{\underline{i}} R_{i}\right)=F(\underbrace{\wedge}_{i \in \Lambda} R_{i}\rangle\right)=\overline{\left\langle\hat{i \in \Lambda}_{i} R_{i}\right\rangle}=\overline{\left[\hat{[i \in \Lambda}_{i} R_{i}\right]} \\
& =\wedge_{i \in \Lambda} \overline{R_{i}}=\wedge_{i \in \Lambda} F\left(\underline{R_{i}}\right) .
\end{aligned}
$$

So, the mapping F: $\mathbf{R L A O}(\mathbf{X}) \longrightarrow \mathbf{R U A O}(\mathbf{X})$ preserves arbitrary intersection.

\section{Conclusions}

Following the idea of $L$-fuzzy generalized neighborhood systems as introduced by Zhao et al., we gave the lattice structures of lower and upper approximation operators based on L-fuzzy generalized neighborhood systems. In particular,

(1) If $L$ satisfies the double negative law, then the joincomplete lattices $(\mathbf{L A O}(\mathbf{X}), \leq, \mathrm{V})$ and $(\mathbf{U A O}(\mathbf{X}), \leq, \vee)$ are order isomorphic (here $\mathbf{L A O}(\mathbf{X})$ and $\mathbf{U A O}(\mathbf{X})$ are the family of all lower and upper approximation operators based on $L$-fuzzy generalized neighborhood systems, respectively), including when $L$-fuzzy generalized neighborhood system is serial, reflexive, and transitive.

(2) If $L$ satisfies the double negative law, then the complete lattices $(\boldsymbol{R L A O}(\mathbf{X}), \leq, \vee, \wedge)$ and $(\mathbf{R U A O}(\mathbf{X}), \leq, \vee, \wedge)$ are complete lattice isomorphism (here $\operatorname{RLAO}(\mathbf{X})$ and $\operatorname{RUAO}(\mathbf{X})$ are the family of all lower and upper approximation operators based on $L$-fuzzy relations, respectively).

\section{Data Availability}

No data were used to support this study.

\section{Conflicts of Interest}

The authors declare that they have no conflicts of interest.

\section{Acknowledgments}

This study was partly supported by the National Natural Science Foundation of China (grant nos. 11771263, 11671007, and 61976130), the Applied Basic Research
Program Funded by Qinghai Province (program no. 2019ZJ-7078), the Scientific Research Program Funded by Shaanxi Provincial Education Department (program no. 18JK0360), and the Doctoral Scientific Research Foundation of Xi'an Polytechnic University (grant no. BS1426).

\section{References}

[1] Z. Pawlak, "Rough sets," International Journal of Computer \& Information Sciences, vol. 11, no. 5, pp. 341-356, Oct. 1982.

[2] Z. Pawlak, "Rough sets and fuzzy sets," Fuzzy Sets and Systems, vol. 17, no. 1, pp. 99-102, Sep. 1985.

[3] T. Herawan, M. M. Deris, and J. H. Abawajy, "A rough set approach for selecting clustering attribute," Knowledge-Based Systems, vol. 23, no. 3, pp. 220-231, Apr. 2010.

[4] J. Hao and Q. Li, "The relationship between L-fuzzy rough set and L-topology," Fuzzy Sets and Systems, vol. 178, no. 1, pp. 74-83, 2011.

[5] K. Y. Huang, T.-H. Chang, and T.-C. Chang, "Determination of the threshold value $\beta$ of variable precision rough set by fuzzy algorithms," International Journal of Approximate Reasoning, vol. 52, no. 7, pp. 1056-1072, Oct. 2011.

[6] L. Q. Li, Q. Jin, and K. Hu, "Lattice-valued convergence associated with cns spaces," Fuzzy Sets And Systems, vol. 370, 2018.

[7] G. Liu and W. Zhu, "The algebraic structures of generalized rough set theory," Information Sciences, vol. 178, no. 21, pp. 4105-4113, 2008.

[8] W. Wu and W. X. Zhang, "Constructive and axiomatic approaches of fuzzy approximation operators," Information Sciences, vol. 159, no. 3-4, pp. 233-254, 2004.

[9] D. S. Yeung, D. G. Degang Chen, E. C. C. Tsang, J. W. T. Lee, and X. Z. Wang Xizhao, "On the generalization of fuzzy rough sets," IEEE Transactions on Fuzzy Systems, vol. 13, no. 3, pp. 343-361, 2005.

[10] H. Zhang, "Two new operators in rough set theory with applications to fuzzy sets," Information Sciences, vol. 166, no. 1-4, pp. 147-165, 2004.

[11] J. B. Michael and T. Y. Lin, Neighborhoods, Rough Sets and Query Relaxation in Cooperative Answering," Rough Sets and Data Mining: Analysis of Imprecise Data, Kluwer Academic Publisher, Dordrecht, Netherland, 1997.

[12] W. Wu, J. S. Mi, and W. X. Zhang, "Generalized fuzzy rough sets," Information Sciences, vol. 151, pp. 263-282, 2003.

[13] X. Yang and T. Li, "The minimization of axiom sets characterizing generalized approximation operators," Information Sciences, vol. 176, no. 7, pp. 887-899, 2006.

[14] W. Zhu, "Generalized rough sets based on relations," Information Sciences, vol. 177, no. 22, pp. 4997-5011, 2007.

[15] T. Deng, Y. Chen, W. Xu, and Q. Dai, "A novel approach to fuzzy rough sets based on a fuzzy coverings," Information Sciences, vol. 177, no. 11, pp. 2308-2326, 2007.

[16] Y. Yao and B. Yao, "Covering based rough set approximations," Information Sciences, vol. 200, pp. 91-107, 2012.

[17] Y.-L. Zhang, C.-Q. Li, M.-L. Lin, and Y.-J. Lin, "Relationships between generalized rough sets based on covering and reflexive neighborhood system," Information Sciences, vol. 319, pp. 56-67, 2015.

[18] W. Zhu, "Topological approaches to covering rough sets," Information Sciences, vol. 177, no. 6, pp. 1499-1508, 2007.

[19] W. Zhu, "Relationship between generalized rough sets based on binary relation and covering," Information Sciences, vol. 179, no. 3, pp. 210-225, 2009. 
[20] R. Bělohlávek, "Fuzzy closure operators II: induced relations, representation, and examples," Soft Computing, vol. 7, no. 1, pp. 53-64, 2002.

[21] S. E. Han, L. X. Lu, and W. Yao, "Quantale-valued fuzzy scott topology," Iranian Journal of Fuzzy Systems, vol. 16, no. 3, pp. 175-188, 2019.

[22] Q. Jin and L. Li, "One-axiom characterizations on latticevalued closure (interior) operators," Journal of Intelligent \& Fuzzy Systems, vol. 31, no. 3, pp. 1679-1688, 2016.

[23] Q. Jin and L. Li, "Modified Top-convergence spaces and their relationships to lattice-valued convergence spaces," Journal of Intelligent \& Fuzzy Systems, vol. 35, no. 2, pp. 2537-2546, 2018.

[24] Q. Jin, L. Li, Y. Lv, F. Zhao, and J. Zou, "Connectedness for lattice-valued subsets in lattice-valued convergence spaces," Quaestiones Mathematicae, vol. 42, no. 2, pp. 135-150, 2019.

[25] H. Lai and D. Zhang, "Fuzzy topological spaces with conical neighborhood systems," Fuzzy Sets and Systems, vol. 330, pp. 87-104, 2018.

[26] L. Li, "p-Topologicalness-A relative topologicalness in T-Convergence spaces," Mathematics, vol. 7, no. 3, p. 228, 2019.

[27] L. Li and Q. Jin, "On adjunctions between Lim, SL-Top, and SL-Lim," Fuzzy Sets and Systems, vol. 182, no. 1, pp. 66-78, 2011.

[28] L. Li and Q. Li, "On enriched L-topologies: base and subbase," Journal of Intelligent \& Fuzzy Systems, vol. 28, no. 6, pp. 2423-2432, 2015.

[29] B. Pang and F.-G. Shi, "Subcategories of the category of L-convex spaces," Fuzzy Sets and Systems, vol. 313, pp. 61-74, 2017.

[30] B. Pang and Z.-Y. Xiu, "Stratified L-prefilter convergence structures in stratified L-topological spaces," Soft Computing, vol. 22, no. 22, pp. 7539-7551, 2018.

[31] S. E. Rodabaugh and E. P. Klement, "Topological and Algebraic Structures in Fuzzy Sets," the Handbook of Recent Developments in the Mathematics of Fuzzy Sets, Kluwer Academic Publishers, Dordrecht, Netherland, 2003.

[32] X. Chen and Q. Li, "Construction of rough approximations in fuzzy setting," Fuzzy Sets and Systems, vol. 158, no. 23, pp. 2641-2653, 2007.

[33] L. Q. Li, Q. Jin, K. Hu, and F. F. Zhao, "The axiomatic characterizations onL-fuzzy covering-based approximation operators," International Journal of General Systems, vol. 46, no. 4, pp. 332-353, 2017.

[34] T.-J. Li, Y. Leung, and W.-X. Zhang, "Generalized fuzzy rough approximation operators based on fuzzy coverings," International Journal of Approximate Reasoning, vol. 48, no. 3, pp. 836-856, 2008.

[35] Z. M. Ma and B. Q. Hu, "Topological and lattice structures of -fuzzy rough sets determined by lower and upper sets," Information Sciences, vol. 218, pp. 194-204, 2013.

[36] A. M. Radzikowska and E. E. Kerre, "Fuzzy rough sets based on residuated lattices," Transactions on Rough Sets II, vol. 3135, pp. 278-296, 2004.

[37] Y.-H. She and G.-J. Wang, "An axiomatic approach of fuzzy rough sets based on residuated lattices," Computers \& Mathematics with Applications, vol. 58, no. 1, pp. 189-201, 2009.

[38] S. Sun, L. Q. Li, K. Hu, and A. Ramadan, "L-fuzzy upper approximation operators associated with L-generalized fuzzy remote neighborhood systems of L-fuzzy points," AIMS Mathematics, vol. 5, no. 6, pp. 5638-5652, 2020.
[39] J. A. Goguen, "L-fuzzy sets," Journal of Mathematical Analysis and Applications, vol. 18, no. 1, pp. 145-174, 1967.

[40] A. A. Ramadan, E. H. Elkordy, and M. El-Dardery, " $L$-fuzzy approximation spaces and $L$-fuzzy topological spaces," International Journal of Fuzzy Systems, vol. 13, no. 1, pp. 115129, 2016.

[41] A. A. Ramadan and L. Q. Li, "Categories of lattice-valued closure (interior) operators and Alexandroff L-fuzzy topologies," International Journal of Fuzzy Systems, vol. 16, pp. 73-84, 2019.

[42] Z. Wang, Y. Wang, and K. Tang, "Some properties of L-fuzzy approximation spaces based on bounded integral residuated lattices," Information Sciences, vol. 278, pp. 110-126, 2014.

[43] W.-Z. Wu, Y. Leung, and J.-S. Mi, "On characterizations of -fuzzy rough approximation operators," Fuzzy Sets and Systems, vol. 154, no. 1, pp. 76-102, 2005.

[44] W. Yao and B. Zhao, "Kernel systems on L-ordered sets," Fuzzy Sets and Systems, vol. 182, no. 1, pp. 101-109, 2011.

[45] Y. Yao, "Neighborhood systems and approximate retrieval," Information Sciences, vol. 176, no. 23, pp. 3431-3452, 2006.

[46] J. Zhan, B. Sun, and J. C. R. Alcantud, "Covering based multigranulation(I,T)-fuzzy rough set models and applications in multi-attribute group decision-making," Information Sciences, vol. 476, pp. 290-318, 2019.

[47] T. Y. Lin, "Neighborhood systems: a qualitative theory for fuzzy and rough sets," Advances in Machine Intelligence and Soft Computing, vol. 4, pp. 132-155, 1997.

[48] Y.-R. Syau and E.-B. Lin, "Neighborhood systems and covering approximation spaces," Knowledge-Based Systems, vol. 66, pp. 61-67, 2014.

[49] F. Zhao and L. Li, "Axiomatization on generalized neighborhood system-based rough sets," Soft Computing, vol. 22, no. 18, pp. 6099-6110, 2018.

[50] F. F. Zhao, L. Q. Li, S. B. Sun, and Q. Jin, "Rough approximation operators based on quantale-valued fuzzy generalized neighborhood systems," Iranian Journal of Fuzzy Systems, vol. 16, no. 6, pp. 53-63, 2019.

[51] E. Bartl and M. Krupka, "Residuated lattices of block relations: size reduction of concept lattices," International Journal of General Systems, vol. 45, no. 7-8, pp. 773-789, 2016.

[52] R. Bělohlávek, Fuzzy Relational Systems, Foundations and Principles, Kluwer Academic Publishers, New York, NY, USA, 2002.

[53] P. Hájek, Metamathematics of Fuzzy Logic, Kluwer Academic Publishers, Dordrecht, Netherland, 1998. 